International Journal of Pure and Applied Mathematics

Volume 83 No. 3 2013, 387-395

ISSN: 1311-8080 (printed version); ISSN: 1314-3395 (on-line version)

url: http://www.ijpam.eu

doi: http://dx.doi.org/10.12732/ijpam.v83i3.1

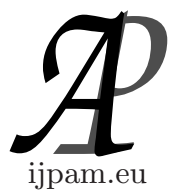

\title{
A COLLOCATION METHOD WITH RBF FOR PDE ON INFINITE DOMAIN
}

\author{
M. Moini \\ Department of Mathematics \\ Roudehen Branch \\ Islamic Azad University \\ Roudehen, IRAN
}

\begin{abstract}
This paper is concerned with the numerical solution of PDEs problem on an infinite domain. An artificial boundary is introduced to make the computational domain finite. On the artificial boundary an exact boundary condition is applied to reduce the original problem to an initial boundary value problem. The collocation method with a radial basis functions is used that approximates the solution of converted problem. Two numerical examples are performed to show the accuracy of the scheme.
\end{abstract}

\section{AMS Subject Classification: $35 \mathrm{~F} 16$}

Key Words: infinite domain, artificial boundary condition, collocation method, radial basis function

\section{Introduction}

As is well known, many application problems can be model by parabolic equation on infinite domains, such as the heat transfer problem, fluid dynamics problems, and option pricing problems. For some problems, the analytic solu- 
tion can be obtained, and it is still an effective way to solve the problem. When the analytic solution is not available, or the analytic solution is too complicated to use, then a numerical method is necessary for solving the problem. The numerical method for PDE problems should be high-order accurate, flexible with respect to the geometry, computationally efficient, and easy to implement. The methods that are commonly used, usually fulfill one or two of the criteria, but not all. Finite difference methods can be made high-order accurate, but require a structured grid. Spectral methods are even more accurate, but have severe restrictions on the geometry.

A fairly new approach to solving PDEs is through radial basis functions (RBFs). A key feature of an RBF method is that it does not require a grid. The only geometric properties that are used in an RBF approximation are the pairwise distances between points. Distances are easy to compute in any number of space dimensions, so working in higher dimensions does not increase the difficulty. In this paper, we study the numerical solution of PDEs problem on infinite domain. In order to solve this problem by numerical methods, one has to consider a finite subdomain and impose an artificial boundary condition. When the solution of this new problem is equal to the restriction to the subdomain of the original solution, we say that the artificial boundary condition is transparent. Here, we apply the collocation method with RBFs to solve one dimensional time dependent PDEs problem with transparent boundary condition. we consider an algorithm consist of three parts, part one, two and three are related to variables $t, x$ and $t$ respectively. The first part consists of Laplace transform method in time variable, converting the problem to a one parametric Sturm-Liouville equation with Robin's boundary conditions. The collocation method with a RBFs is used in the second part. The last part utilizes Talbot's method for numerical inversion of the Laplace transformation and generates solution for the transformed problem.

The layout of the paper is as follows. In Section 2, we introduce and study one dimensional time dependent PDE problem with TBCs and it's corresponding transformed equations. We given some basic knowledge about RBFs in Section 3. In Section 4, we apply the collocation method for approximating numerical solution of PDE with RBFs. The results of numerical experiments are presented in Section 5. 


\section{The Construction of the Model}

We consider the following initial value problem of PDE [1] on $R^{1} \times[0, T]$ :

$$
\begin{gathered}
\alpha u_{t}(x, t)=\beta u_{x x}(x, t)+\gamma u(x, t), \quad \forall(x, t) \in R^{1} \times(0, T], \\
u(x, 0)=u^{0}(x), \quad \forall x \in R^{1},
\end{gathered}
$$

where $u^{0}(x)$ is the initial data given on $R^{1}$, the unknown function $u(x, t)$ is a complex value function on $R^{1} \times(0, T]$ and $\alpha, \beta, \gamma$ are constant.

In order to solve such whole-space problems by numerical methods, one has to consider a finite subdomain and impose an artificial boundary condition. Suppose that $u^{0}$ is compact with $\operatorname{supp}\left\{u^{0}\right\} \subset[0,1]$.

Then, we can introduce two artificial boundaries $\Gamma_{0}=\{(x, t) \mid x=0, \quad 0<$ $t \leq T\}$ and $\Gamma_{1}=\{(x, t) \mid x=1, \quad 0<t \leq T\}$, which divide $R^{1} \times[0, T]$ into three parts, $\Omega_{0}=\{(x, t) \mid-\infty<x \leq 0,0<t \leq T\}, \Omega_{1}=\{(x, t) \mid 1 \leq x<$ $+\infty, 0<t \leq T\}, \Omega^{c}=\{(x, t) \mid 0<x<1,0<t \leq T\}$. The finite subdomain $\Omega^{c}$ is our computational domain. Transparent boundary conditions are nonlocal in $t$ and read:

$$
\begin{array}{ll}
u_{x}(0, t)=k_{0} \frac{d}{d t} \int_{0}^{t} \frac{u(0, \lambda)}{\sqrt{t-\lambda}} d \lambda, & \text { on } \Gamma_{0}, \\
u_{x}(1, t)=k_{1} \frac{d}{d t} \int_{0}^{t} \frac{u(1, \lambda)}{\sqrt{t-\lambda}} d \lambda, & \text { on } \Gamma_{1} .
\end{array}
$$

Therefore, the boundary value problem to approximate is now given by:

$$
\begin{gathered}
\alpha u_{t}(x, t)=\beta u_{x x}(x, t)+\gamma u(x, t), \quad \forall(x, t) \in \Omega^{c}, \\
u_{x}(0, t)=k_{0} \frac{d}{d t} \int_{0}^{t} \frac{u(0, \lambda)}{\sqrt{t-\lambda}} d \lambda, \quad 0<t \leq T, \\
u_{x}(1, t)=k_{1} \frac{d}{d t} \int_{0}^{t} \frac{u(1, \lambda)}{\sqrt{t-\lambda}} d \lambda, \quad 0<t \leq T, \\
u(x, 0)=u^{0}(x), \quad 0 \leq x \leq 1 .
\end{gathered}
$$

This initial boundary value problem is well-posed and its solution coincides with the solution of the original problem (1) restricted to $\bar{\Omega}^{c}$ [2]. Here, we focus on the model for bounderies. We consider $u(0,0)=u(1,0)=0$ and let,

$$
\frac{d}{d t} \int_{0}^{t} \frac{u(0, \lambda)}{\sqrt{t-\lambda}} d \lambda=2 \frac{d}{d t} \int_{0}^{t} \sqrt{t-\lambda} \frac{d}{d \lambda}(u(0, \lambda) d \lambda)
$$


using Liebnitz differentiation formula we have:

$$
\frac{d}{d t} \int_{0}^{t} \frac{u(0, \lambda)}{\sqrt{t-\lambda}} d \lambda=\int_{0}^{t} \frac{d}{d \lambda}\{u(0, \lambda)\} \frac{d \lambda}{\sqrt{t-\lambda}} .
$$

According to $\mathrm{Eq}(7)$, we can rewrite Eqs.(5) and(6) as follows:

$$
\begin{aligned}
& u_{x}(0, t)=k_{0} \int_{0}^{t} \frac{d}{d \lambda}\{u(0, \lambda)\} \frac{d \lambda}{\sqrt{t-\lambda}}, \\
& u_{x}(1, t)=k_{1} \int_{0}^{t} \frac{d}{d \lambda}\{u(1, \lambda)\} \frac{d \lambda}{\sqrt{t-\lambda}} .
\end{aligned}
$$

We apply the Laplace transform to Eqs. (4),(8)and(9), we have:

$$
\begin{gathered}
\alpha s U(x, s)-\alpha u(x, 0)=\beta U_{x x}(x, s)+\gamma U(x, s), \quad \forall(x, s) \in \Omega^{e}, \\
U_{x}(0, s)=k_{0} s \sqrt{\frac{\pi}{s}} \cdot U(0, s), \quad \delta_{0}<s<\infty, \\
U_{x}(1, s)=k_{1} s \sqrt{\frac{\pi}{s}} \cdot U(1, s), \quad \delta_{0}<s<\infty .
\end{gathered}
$$

where $U$ is Laplace transform of $u$ and

$$
\Omega^{e}=\left\{(x, s) \mid \quad 0<x<1, \quad \delta_{0}<s<\infty\right\} .
$$

For discrete, we consider $h=\frac{1}{N}$, where $N$ is a positive integer and $h$ is spatial size. The one dimensional nodal points are defined as $x_{i}=i h, \quad i=$ $0,1,2, \ldots, N$.

\section{Basic Knowledge about RBF}

The approximation of a distribution $u(x)$, using RBFs, may be written as a linear combination of $N$ radial functions, usually it takes the following form[6]:

$$
\tilde{u}(x)=\sum_{i=1}^{N} \lambda_{i} \phi\left(x, x_{i}\right)+\psi(x) \quad, x \in \Omega \subset R^{d},
$$

where $N$ is the number of data points, $x=\left(x_{1}, x_{2}, \ldots, x_{d}\right), d$ is the dimension of the problem, the $\lambda_{i}(i=1,2, \ldots, N)$ are coefficients to be determined, $\phi$ is the $\mathrm{RBF}$ and $\psi$ is the additional polynomial. $\tilde{u}(x)$ can be written without the $\psi$. The commonly RBFs are as follows: 


$$
\begin{aligned}
& \text { (MQ): } \phi\left(x, x_{i}\right)=\phi\left(r_{i}\right)=\left(r^{2}+c^{2}\right)^{\frac{\xi}{2}}, \xi=1, \ldots, 2 N+1, \ldots, \\
& \text { (IMQ): } \phi\left(x, x_{i}\right)=\phi\left(r_{i}\right)=\left(r^{2}+c^{2}\right)^{\frac{\xi}{2}},-\xi=1, \ldots, 2 N+1, \ldots \\
& \text { Gaussian: } \phi(r)=e^{-c^{2} r^{2}}
\end{aligned}
$$

where $r_{i}=\left\|x-x_{i}\right\|$ is the usual Euclidean norm and $c$ is a positive parameter, respectively.

The convergence proofs in applying the RBFs for scattered data interpolation was given by $\mathrm{Wu}[7]$.

\section{The RBF Collocation Method}

The approximation of the $u(x, t)$, using RBFs, may be written as a linear combination of $N$ radial functions, usually it takes th following form[3]:

$$
\tilde{u}(x, t)=\sum_{i=1}^{N-1} q_{i}(t) \phi_{i}(x)+p(x),
$$

where $q_{i}(t)(i=1, \ldots, N-1)$ are unknown coefficients to be determined and $p(x)$ is a polynomial of first degree corresponding with the $x_{0}$ and $x_{N}$ points.

We consider (13) as follows:

$$
\tilde{u}(x, t)=\sum_{i=0}^{N} q_{i}(t) \phi_{i}(x),
$$

where:

$$
\phi_{i}(x)= \begin{cases}p(x) & \text { for } \quad i=0, N \\ \phi_{i}(x) & \text { for } \quad i=1, \ldots, N-1,\end{cases}
$$

$q_{0}(t)=q_{i}(N)=$ constant.

$\mathrm{Eq}(14)$ can be written in matrix form as: $\quad \tilde{u}(x, t)=\Phi(x) \mathbf{q}(t)$, where:

$$
\Phi(x)=\left[\phi_{0}(x), \phi_{1}(x), \ldots, \phi_{N}(x)\right], \quad \mathbf{q}(t)=\left[q_{0}(t), q_{1}(t), \ldots, q_{N}(t)\right]^{T} .
$$

Collocating interpolation model (14)at the $N+1$ nodal points, lead to the system of equations $\Phi_{B} \mathbf{q}=\tilde{\mathbf{u}}$ where $\tilde{\mathbf{u}}=\left[\tilde{u}_{0}, \tilde{u}_{1}, \ldots, \tilde{u}_{N}\right]$,

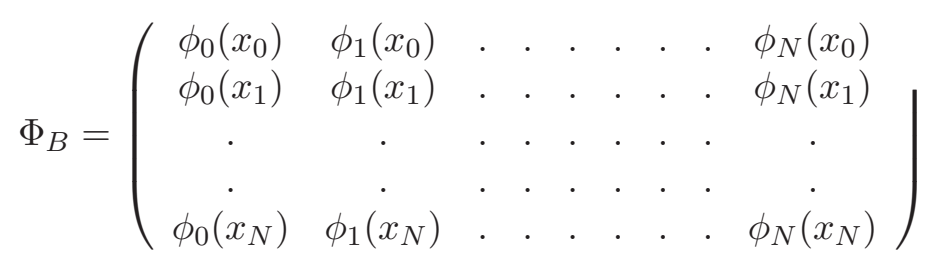


Here $\tilde{\mathbf{u}}\left(\tilde{u}_{i}, i=0,1,2, \ldots N\right)$ are the nodal values of $\tilde{u}$ at the $N+1$ collocation points. Note that $\Phi_{B}$ is an $(N+1) \times(N+1)$ matrix. Laplace transform of $(14)$ is as follows:

$$
\tilde{U}(x, s)=\sum_{i=0}^{N} Q_{i}(s) \phi_{i}(x)
$$

where $Q$ is Laplace transform of $q$. Substituting (14),(15)into (10),(11),(12), we can obtain the following schemes:

$$
\begin{array}{r}
\alpha s \sum_{i=0}^{N} Q_{i}(s) \phi_{i}\left(x_{k}\right)-\alpha \sum_{i=0}^{N} q_{i}(0) \phi_{i}\left(x_{k}\right)=\beta \sum_{i=0}^{N} Q_{i}(s) \phi_{i}^{\prime \prime}\left(x_{k}\right)+ \\
\gamma \sum_{i=0}^{N} Q_{i}(s) \phi_{i}\left(x_{k}\right), \quad k=1, \ldots, N-1, \quad \delta_{0}<s<\infty, \\
\sum_{i=0}^{N} Q_{i}(s) \phi_{i}^{\prime}(0)=k_{0} s \sqrt{\frac{\pi}{s}} \sum_{i=0}^{N} Q_{i}(s) \phi_{i}(0), \quad \delta_{0}<s<\infty, \\
\sum_{i=0}^{N} Q_{i}(s) \phi_{i}^{\prime}(1)=k_{1} s \sqrt{\frac{\pi}{s}} \sum_{i=0}^{N} Q_{i}(s) \phi_{i}(1), \quad \delta_{0}<s<\infty .
\end{array}
$$

Hence we have to solve the following $(N+1) \times(N+1)$ linear algebraic system:

$$
\begin{gathered}
\Phi^{\prime}(0) Q(s)-k_{0} s \sqrt{\frac{\pi}{s}} \Phi(0) Q(s)=0, \\
(\alpha s-\gamma) \bar{\Phi}_{B} Q(s)-\beta \bar{\Phi}_{B}^{\prime \prime} Q(s)=\alpha \bar{\Phi}_{B} q(0), \\
\Phi^{\prime}(1) Q(s)-k_{1} s \sqrt{\frac{\pi}{s}} \Phi(1) Q(s)=0,
\end{gathered}
$$

for the unknowns $Q(s)$, where $Q(s)=\left[Q_{0}(s), Q_{1}(s), \ldots, Q_{N}(s)\right]^{T}, \bar{\Phi}_{B}$ is the matrix that obtained with omission the first and the last rows from matrix $\Phi_{B}$. Uniqueness of the solution $(16),(17),(18)$ is a consequence of the following theorem.

Theorem 1. If $f$ is completely monotone but not constant on $[0, \infty]$, then the function $x \longrightarrow f\left(\|x\|^{2}\right)$ is a radial, strictly positive definite function on any inner-product space. Thus, for any $n$ distinct points $x_{1}, x_{2}, \ldots, x_{n}$ in such a space the matrix $A_{i j}=f\left(\left\|x_{i}-x_{j}\right\|^{2}\right)$ is positive definite (and therefore nonsingular), see [4]. 
We can obtain the corresponding system of (16), (17), (18) which involves $(N+$ $1)$ equations and $(N+1)$ unknowns. This system can be solved using either a direct method or iteration methods. Here, we use Talbot's method [5] to approximation inversion of Laplace transform.

\section{Numerical Example}

In this section we consider two examples. In each example, for numerical implementation of collocation method we use global radial base functions MQ with $\zeta=1$, IMQ with $\zeta=-1$ and Gaussian.

Tables 1 and 2 show error of numerical solutions for original functions $u, \tilde{u}$ and transformed functions $U, \tilde{U}$ on the set of nodal points $R_{M}=\left\{\left(\xi_{i}, s_{j}\right) \mid 0 \leq\right.$ $i, j \leq M\}$, where $\left(\xi_{i}, s_{j}\right) \in[0,1] \times[\underline{s}, \bar{s}]$ are two dimensional random nodes. We take number of nodes $N$, precision $=15.9546, \underline{s}=\operatorname{mins}=1$ and $\bar{s}=\operatorname{maxs}=$ 1000.

Example 1. We consider the following sample problem:

$$
\begin{gathered}
u_{t}(x, t)=u_{x x}(x, t)+u(x, t)+x(1-x)+t\left(2-x+x^{2}\right), 0 \leq x \leq 1,0<t \leq 1 \\
u_{x}(0, t)=\int_{0}^{t} \frac{d u(0, \lambda)}{d \lambda} \cdot \frac{1}{\sqrt{t-\lambda}} d \lambda+t, \quad 0<t \leq 1, \\
u_{x}(1, t)=2 \int_{0}^{t} \frac{d u(1, \lambda)}{d \lambda} \cdot \frac{1}{\sqrt{t-\lambda}} d \lambda-t, \quad 0<t \leq 1, \\
u(x, 0)=0, \quad 0 \leq x \leq 1 .
\end{gathered}
$$

Numerical results of Example 1 are given in Table 1:

The analytic solution is given by: $u(x, t)=t(1-x) x$.

Example 2. We consider sample problem as follows:

$$
\begin{gathered}
u_{t}(x, t)=u_{x x}(x, t)+u(x, t)+e^{-t}\left((1-x) x+2 t\left(1-x+x^{2}\right)\right), \\
0 \leq x \leq 1,0<t \leq 1 \\
u_{x}(0, t)=\int_{0}^{t} \frac{d u(0, \lambda)}{d \lambda} \cdot \frac{1}{\sqrt{t-\lambda}} d \lambda+t e^{-t}, \quad 0<t \leq 1,
\end{gathered}
$$




\begin{tabular}{|c|c|c|c|c|}
\hline RBF & $\mathrm{N}$ & $\mathrm{c}$ & $\|\tilde{U}-U\|_{\infty}$ & $\|\tilde{u}-u\|_{\infty}$ \\
\hline MQ & 9 & 1 & $1.6 \times 10^{-5}$ & $8.4 \times 10^{-5}$ \\
IMQ & 9 & 1 & $6.30 \times 10^{-4}$ & $5.55 \times 10^{-4}$ \\
Gaussian & 9 & 0.4 & $1.96 \times 10^{-4}$ & $7.04 \times 10^{-4}$ \\
\hline MQ & 11 & 1 & $2.53 \times 10^{-5}$ & $1.64 \times 10^{-5}$ \\
IMQ & 11 & 1 & $1.69 \times 10^{-4}$ & $9.09 \times 10^{-5}$ \\
Gaussian & 11 & 0.42 & $7.43 \times 10^{-4}$ & $4.47 \times 10^{-4}$ \\
\hline
\end{tabular}

Table 1

$$
\begin{gathered}
u_{x}(1, t)=2 \int_{0}^{t} \frac{d u(1, \lambda)}{d \lambda} \cdot \frac{1}{\sqrt{t-\lambda}} d \lambda-t e^{-t}, \quad 0<t \leq 1 \\
u(x, 0)=0, \quad 0 \leq x \leq 1 .
\end{gathered}
$$

And the analytic solution is given by: $u(x, t)=t e^{-t}(1-x) x$.

Numerical results of Example 2 are given in Table 2:

\begin{tabular}{|c|c|c|c|c|}
\hline RBF & $\mathrm{N}$ & $\mathrm{c}$ & $\|U-U\|_{\infty}$ & $\|\tilde{u}-u\|_{\infty}$ \\
\hline MQ & 9 & 1 & $3.56 \times 10^{-5}$ & $4.58 \times 10^{-5}$ \\
IMQ & 9 & 1 & $2.09 \times 10^{-4}$ & $2.69 \times 10^{-4}$ \\
Gaussian & 9 & 0.56 & $1.90 \times 10^{-4}$ & $2.45 \times 10^{-4}$ \\
\hline MQ & 11 & 1 & $6.32 \times 10^{-6}$ & $8.14 \times 10^{-6}$ \\
IMQ & 11 & 1 & $4.24 \times 10^{-5}$ & $5.46 \times 10^{-5}$ \\
Gaussian & 11 & 1 & $8.38 \times 10^{-8}$ & $1.13 \times 10^{-7}$ \\
\hline
\end{tabular}

Table 2

\section{References}

[1] H. Han, J. Jin, X. Wu, A finite-difference method for the one-dimensional time-dependent schrodinger equation on unbounded domain, Computers and Mathematics with Applications, 50 (2005), 1345-1362.

[2] X. Antoine, C. Besse, Unconditionally stable discretization schemes of nonreflecting boundary conditions for the one-dimensional Schrodinger equation, J. Comput. Phys., 188 (2003), 157-175. 
[3] P. Reuter, I. Tobor, C. Schlick, S. Dedieu, Point-based modelling and Rendering using radial basis functions, In: Proceedings of the 1-st International Conference on Computer Graphics and Interactive Techniques in Australasia and South East Asia (2003), 111-118.

[4] E.W. Cheney, A Course in Proximation Theory, Brooks/Cole Publishing Company (1999).

[5] A. Murli, M. Rizzardi, Talbot's method for the laplace inversion problem, ACM Transactions on Mathematical Software, 16, No. 2 (1990), 158-168.

[6] E.J. Kansa, Multiquadric scattered data approximation scheme with applications to computational fluid dynamicsП, Comput. Math. Appl., 19 (1990), 147-161.

[7] Z. Wu, R. Schaback, Local error estimates for RBF interpolation of scattered datd, IMA J. Numer. Anal., 13 (1993), 13-27. 
